\title{
Camera Calibration using Vanishing Points
}

\author{
Paul Beardsley and David Murray * \\ Department of Engineering Science, University of Oxford, Oxford OX1 3PJ, UK
}

\begin{abstract}
This paper describes a method for measuring the intrinsic parameters of a camera. If aspect ratio is already known, the method requires two images of a plane; if aspect ratio is not known, the method requires three images of the plane. (Equivalently, one image of $\mathrm{n}$ planes could be used instead of $\mathrm{n}$ images of one plane). The plane must be marked with four points in known configuration.
\end{abstract}

\section{Introduction}

This paper addresses the measurement of a camera's intrinsic parameters, Figure 1. Main references for camera calibration include [7], [4]. The underlying theme in the calibration processing described here is the use of vanishing point and vanishing line information. Related methods are [3], [8], [2].

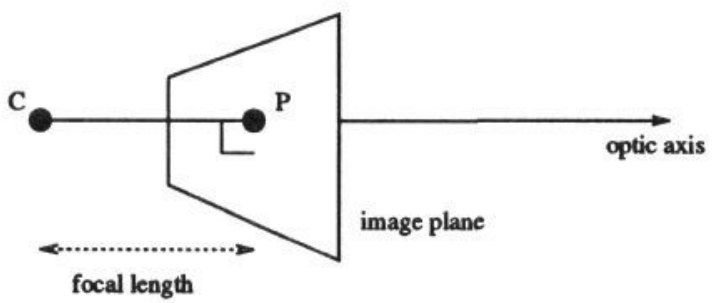

Figure 1: Intrinsic parameters. The camera's focal point is $\mathrm{C}$. The perpendicular projection of $\mathrm{C}$ onto the image plane is the principal point $\mathrm{P}$. The distance $\mathrm{CP}$ is the focal length. On the image plane, the ratio of unit lengths along the $\mathrm{y}-$ and $\mathrm{x}$-axes is the aspect ratio.

Section 2 describes some key concepts used in the calibration, sections 3 to 6 cover the calibration itself, and section 7 contains the results.

\section{Key Concepts}

Some important ideas from projective geometry are reviewed here. See [6] for fuller discussion.

Consider a set of parallel lines on a projective plane, Figure 2 - the lines intersect at infinity, at an ideal point. There is a unique association between parallel lines of a given direction and the corresponding ideal point (in this sense, an ideal point can be regarded as specifying a direction). The set of all the ideal points on a projective plane constitutes an ideal line.

\footnotetext{
*This work was supported by SERC Grant No GR/G30003. PAB is in receipt of a SERC studentship.
} 


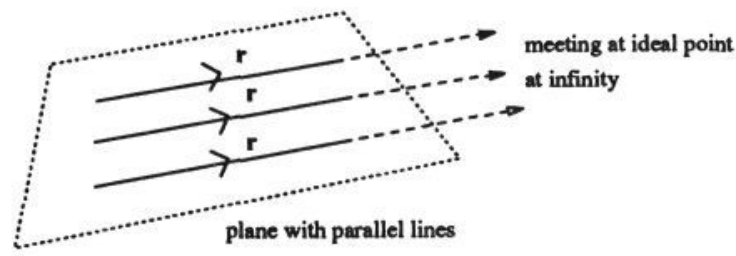

Figure 2: Parallel lines on a projective plane have a well-defined intersection point at infinity, at an ideal point.

The image of an ideal point is a vanishing point, Figure 3 . This figure also shows a standard result from projective geometry - the line $C V$ through the camera focal point and the vanishing point is parallel to the lines in the scene which have given rise to the vanishing point.

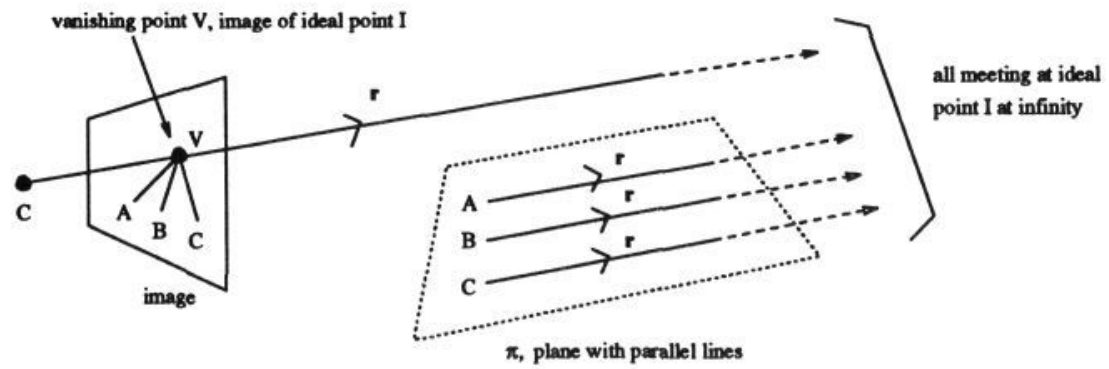

Figure 3: A camera with focal point $\mathrm{C}$ views a set of parallel lines on the plane $\pi$. The parallel lines intersect at the ideal point $\mathrm{I}$. The corresponding image lines converge to the vanishing point $\mathrm{V}$, which is the image of $\mathrm{I}$. Note that the ray $C V$ is parallel to the lines in the scene.

The other main idea in the work concerns the choice of coordinate frame for the processing. Figure 4 shows a set of points $q_{i}$ on the image plane and the corresponding points $Q_{i}$ on a plane $\pi$ in the scene. The projectivity from the image plane to $\pi$ is denoted $\alpha$ and the inverse projectivity from $\pi$ to the image plane is $\alpha^{-1}$. The coordinates of an image point $q_{n}$ and the coordinates of the corresponding scene point $Q_{n}$ are related by $Q_{\mathbf{n}}=\alpha \mathbf{q}_{\mathbf{n}}$ and $\mathbf{q}_{\mathbf{n}}=\alpha^{-1} \mathbf{Q}_{\mathbf{n}}$. This can be treated conceptually as if there is a single point which has coordinates $q_{n}$ in the image coordinate frame, and $\mathbf{Q}_{\mathbf{n}}$ in a coordinate frame based on $\pi$. Thus $\alpha$ and $\alpha^{-1}$ can be regarded as operators which describe a change of coordinate frame. This conceptual treatment is the one adopted for the rest of the paper.

\section{Finding the Principal Point - Overview}

The intrinsic parameters of the camera are found sequentially, starting with the principal point. This section is an overview of the method for finding the principal point, while a step-by-step description is given in Section 4.

Consider a camera with focal point $C$ and principal point $P$ viewing a plane $\pi$. 


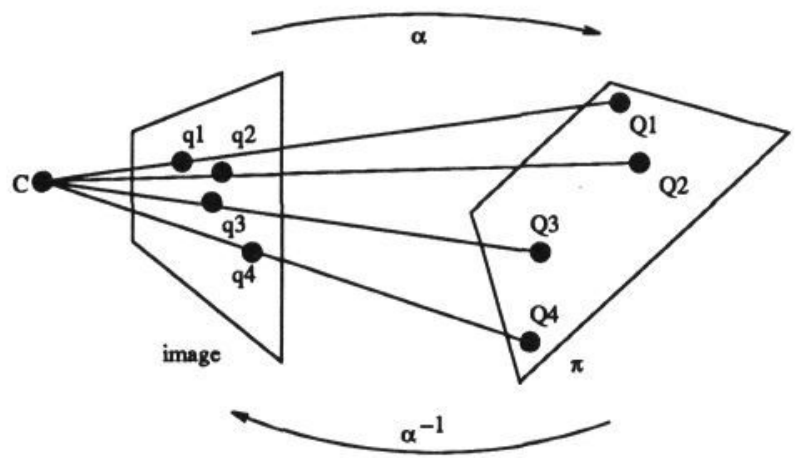

Figure 4: A camera views a plane $\pi$ in the scene. The projectivities between the image plane and $\pi$ are $\alpha$ and $\alpha^{-1}$, in the directions shown. As described in the text, $\alpha$ and $\alpha^{-1}$ can be regarded as operators which transform the coordinates of a point between the image coordinate frame and a coordinate frame based on $\pi$.

The vanishing line of $\pi$ on the image plane is $L$, Figure $5 \mathrm{a}$. The question is whether it is possible to use $L$ to determine the unknown principal point $P$. Clearly, line $L$ on its own is insufficient for this. However, there is a distinguished point on $L$ also shown in Figure 5a - this is the vanishing point $V_{A}$ on $L$ at infinity on the image plane. It will be shown that $V_{A}$ can be used to compute a second vanishing point $V_{B}$ on $L$, Figure $5 \mathrm{~b}$, and the perpendicular to $L$ at $V_{B}$ passes through $P$. Thus, given one image of a plane, the position of $P$ is constrained to lie upon a line $M$; given two or more images of planes in different orientations, the position of $P$ is given by the point of intersection of the set of lines $M_{i}$. (Equivalently, one image of $n$ planes can be used instead of $n$ images of one plane).

(a)

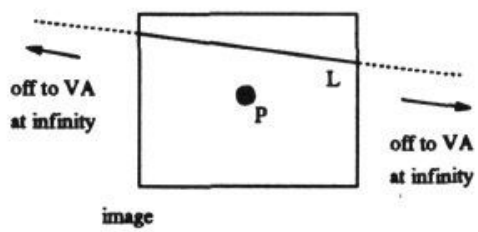

(b)

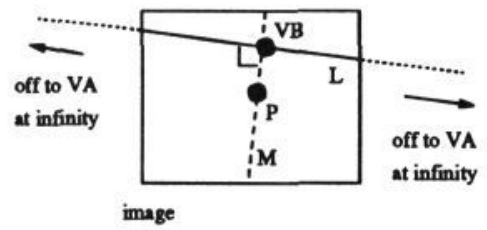

Figure 5: (a) The image contains the principal point $\mathrm{P}$, and the vanishing line $\mathrm{L}$ measured for a plane in the scene. Any point on $\mathrm{L}$ is a vanishing point - the vanishing point which is on $\mathrm{L}$ at infinity on the image plane is $\mathrm{V}_{A}$. (As shown, one can travel to $\mathrm{V}_{A}$ at infinity by going in either direction along $\mathrm{L}$.)

(b) An important vanishing point on $\mathrm{L}$ is $\mathrm{V}_{B}$ which has the property that the perpendicular to $\mathrm{L}$ at $\mathrm{V}_{B}$ passes through the principal point $\mathrm{P}$. 


\section{Finding the Principal Point - Step-By-Step}

(a) Take an image of a plane as shown in Figure 4. Compute the projectivity $\alpha$ from the image plane to $\pi$, and the inverse projectivity $\alpha^{-1}$ from $\pi$ to the image plane. A method for computing a projectivity is given in appendix A. The computation requires four image points, together with the coordinates of the corresponding scene points in a coordinate frame based on $\pi$ (the scale of the configuration of the scene points is immaterial), no three of the four points to be collinear. Apply $\alpha^{\top}$ to the coordinates of the ideal line of $\pi$ in order to obtain the coordinates of the vanishing line $L$ of $\pi$ on the image plane. ${ }^{1}$ See Figure 6.

(b) Find the intersection point $V_{A}$ of $L$ with the ideal line of the image plane.

(c) Apply $\alpha$ to the coordinates of $V_{A}$ to obtain the coordinates of the corresponding ideal point $I_{A}$ on the plane $\pi$.

(d) As pointed out in Section 2, an ideal point such as $I_{A}$ can be associated with a direction on the projective plane. Call the direction $\mathbf{d}_{\mathbf{A}}$. Use $I_{A}$ to compute $I_{B}$ with associated direction $\mathbf{d}_{\mathbf{B}}$, such that $\mathbf{d}_{\mathbf{A}}$ and $\mathbf{d}_{\mathbf{B}}$ are orthogonal. Specifically, if the homogeneous coordinates of $I_{A}$ are $(a, b, 0)$, then a convenient choice for the homogeneous coordinates of $I_{B}$ is $(-b, a, 0)$.

(e) Apply $\alpha^{-1}$ to the coordinates of $I_{B}$ to obtain the coordinates of the corresponding vanishing point $V_{B}$ on the image plane.

To see that $V_{B}$ has the property shown in Figure 5, that the perpendicular to $L$ at $V_{B}$ passes through the principal point $P$, consider the following argument using Figure 6.

(i) Line $L$ is perpendicular to $C P$, which follows from the definition of $P$ (Section 1) and the fact that $L$ lies on the image plane.

(ii) Line $L$ passes through $V_{A}$ and therefore through $I_{A}$ (because $V_{A}$ is the image of $I_{A}$ ). Thus $L$ has direction $\mathrm{d}_{\mathrm{A}}$, the direction associated with $I_{A}$. Line $C V_{B}$ passes through $V_{B}$ and therefore through $I_{B}$ (because $V_{B}$ is the image of $I_{B}$ ). Thus $C V_{B}$ has direction $\mathbf{d}_{\mathbf{B}}$, the direction associated with $I_{B}$. It follows that $L$ is perpendicular to $C V_{B}$.

(iii) The plane $C P V_{B}$ is perpendicular to $L$ because both $C P$ and $C V_{B}$ are perpendicular to $L$. Therefore, any perpendicular to $L$ at $V_{B}$ is in the plane $C P V_{B}$. Therefore, the perpendicular to $L$ at $V_{B}$ which is on the image plane passes through $P$.

In summary, note that a key step in the processing is the generation of the point $V_{B}$ such that the line $C V_{B}$ is perpendicular to $L$. The route to finding $V_{B}$ might seem tortuous. It is necessary because, when working projectively on the image plane, it is not possible to compute a perpendicular such as $L$ and $C V_{B}$ by making use of a point $C$ which is off the image plane. By shifting the coordinate frame to $\pi$, it is possible to bring in the perpendicularity condition using only ideal points which are on the plane $\pi$, and then to transform back to the image plane where the required condition that $L$ is perpendicular to $C V_{B}$ will hold.

\section{Finding the Focal Length}

Figure 7a shows the information available when viewing a plane, following the processing in Section 4 - the vanishing line $L$ of the plane, the special vanishing point

\footnotetext{
${ }^{1} \alpha^{\top}$ is the transpose of $\alpha$. Note that the projectivity $\alpha^{-1}$ between $\pi$ and the image plane is a point projectivity; when operating on a line, as here, the corresponding line projectivity must be used and this is $\alpha^{\top}$. See [6].
} 


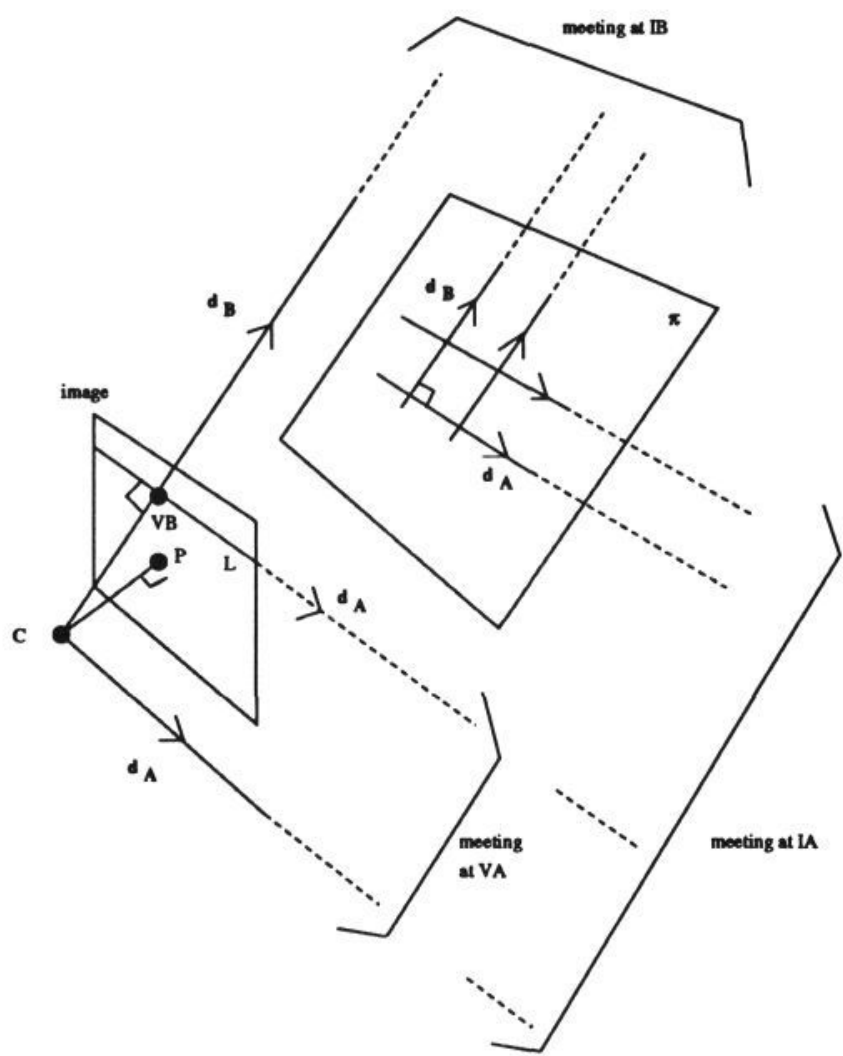

Figure 6: This figure shows all the information involved in computing the vanishing point $\mathrm{V}_{\mathrm{B}}$. First vanishing point $\mathrm{V}_{\mathrm{A}}$ is found. This is the image of the ideal point $\mathrm{I}_{\mathrm{A}}$, so $V_{A}$ can be used to compute the coordinates of $\mathrm{I}_{\mathrm{A}}$. Then $\mathrm{I}_{\mathrm{B}}$ is found using the constraint that the directions associated with $\mathrm{I}_{\mathrm{A}}$ and $\mathrm{I}_{\mathrm{B}}$ are perpendicular. The image of $\mathrm{I}_{\mathrm{B}}$ is $\mathrm{V}_{\mathrm{B}}$, so $\mathrm{I}_{\mathrm{B}}$ can be used to compute the coordinates of $\mathrm{V}_{\mathrm{B}}$.

$V_{B}$ described in Sections 3 and 4 , and the principal point $P$. Figure $7 \mathrm{~b}$ includes one further piece of information, a vanishing point $V_{C}$ lying on $L$ such that angle $V_{B} C V_{C}$ is $45^{\circ}$, to be used in the computation of the focal length.

$V_{C}$ is found by the same method for computing $V_{B}$ from $V_{A}$ in Section 4 - recall the three stages (a) $V_{A}$ was used to compute the coordinates of the corresponding ideal point $I_{A}$, (b) $I_{A}$ was used to compute $I_{B}$ such that the directions associated with $I_{A}$ and $I_{B}$ were perpendicular, and (c) $I_{B}$ was used to compute the coordinates of the corresponding vanishing point $V_{B}$. We apply the same method here to compute $V_{C}$ from $V_{B}$, the only difference lying in the second stage - the ideal point $I_{B}$ is used to compute $I_{C}$ such that the directions associated with $I_{B}$ and $I_{C}$ are at $45^{\circ}$ (this is just as straightforward as computing perpendicular directions).

Once $V_{C}$ is known, the focal length can be found from simple trigonometry. The distance $V_{B} V_{C}$ is known, and this is equal to the distance $C V_{B}$. Thus, the lengths of sides $C V_{B}$ and $V_{B} P$ of the right-angle triangle $C P V_{B}$ are known, and the length $C P$ 
(a)

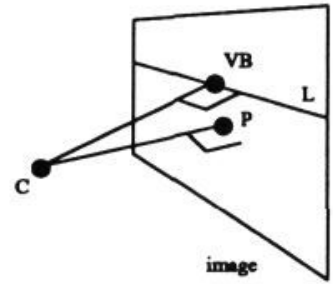

(b)

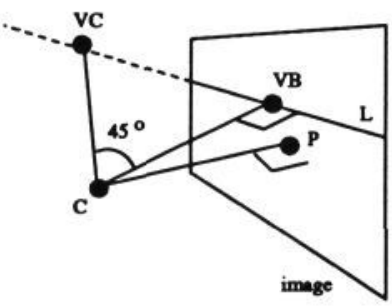

Figure 7: (a) This figure shows just that part of the information in Figure 6 which is to be used in the computation of focal length. (b) One extra piece of information is also used when computing focal length - the point $\mathrm{V}_{C}$ which lies on $\mathrm{L}$ such that angle $\mathrm{V}_{\mathrm{B}} \mathrm{CV}_{\mathrm{C}}$ is $45^{\circ}$.

(which is the focal length) can be computed.

\section{Finding the Aspect Ratio}

It has been assumed so far that the aspect ratio of the image has been corrected. If aspect ratio is unknown, it can be computed in the following way.

Consider the method for computing the principal point in Sections 3 and 4 - a set of "constraint lines" is generated, one from each image, and these lines are concurrent at the principal point. If the aspect ratio has not been corrected, however, the constraint lines will not be concurrent (the constraint lines are generated by finding a perpendicular to the vanishing line $L$, but perpendicularity is not preserved under changes of aspect ratio). Starting from an initial aspect ratio which is near the true value, aspect ratio is iteratively adjusted until the constraint lines are as close as possible to concurrency at this stage, the value of aspect ratio is recorded as its true value. The concurrency test can be carried out given three or more constraint lines.

\section{Results}

Results are given for two cameras which are part of a stereo system. The cameras are both of the same type, so principal point and focal length measurements are expected to be similar, and the aspect ratio is identical. A typical stereo pair of a calibration plane (dimensions about $4 \mathrm{ft}-b y-4 \mathrm{ft}$ ) is shown in Figure 8.

The images are 256-by-256 pixels. The first stage of the processing is to remove radial distortion. The radial distortion correction factor is calibrated separately, by a method [1] which does not require full knowledge of the camera parameters because it assumes approximate values for the radial distortion centre and the aspect ratio ${ }^{2}$. Canny edge detection to sub-pixel accuracy is run on the images, and straight lines are fitted by orthogonal regression along the eight horizontal and eight vertical lines available on the calibration plane. These lines are used to generate 64 vertex positions,

\footnotetext{
${ }^{2}$ It is assumed that the centre of the image array is a reasonable approximation to the radial distortion centre, while a reasonable approximation to the aspect ratio is available from the specification of the digitisation equipment.
} 

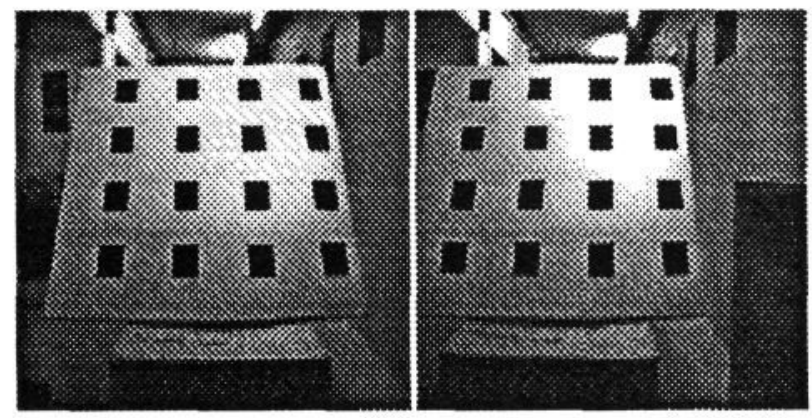

Figure 8: A stereo pair of the calibration plane. (Bowing of straight lines is just discernible - this is due to radial distortion).
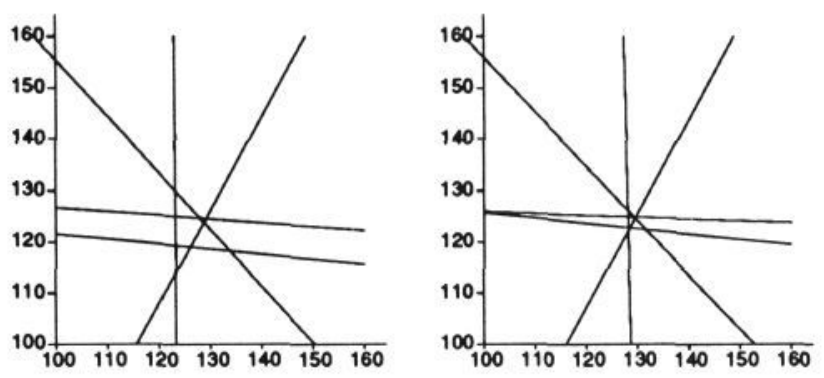

Figure 9: Left and right cameras - the constraint lines meeting at the principal point. The axes are labelled in pixels.

and the vertices are used to determine the projectivity between the image and the calibration plane by the method in appendix A.

Results are shown for five images for the left and right cameras - the calibration plane is repositioned for each image so that the constraint lines used to compute the principal point vary significantly in gradient. Figure 9 shows the five constraint lines meeting at the principal point, when the aspect ratio has been adjusted to its correct value. Table 1 shows the focal lengths computed from the five images. Figure 10 shows the way in which the concurrency of the five constraint lines varies with aspect ratio - the concurrent point of the constraint lines is measured using orthogonal regression, and the residual obtained from this process is used as the measure of concurrency.

Table 2 shows a summary of the results. Obtaining a ground-truth to check camera calibration is difficult - we have so far carried out only one main test. A tape measure is placed parallel to the image plane at a measured distance in front of the camera (the parallelism and the measurement can only be approximate). By comparing the ratio of the tape length to the tape-camera distance, and the ratio of the number of image pixels spanned by the tape to the focal length, it is possible to compute a value for the focal length. The result was within $1 \%$ of the calibrated focal length. This indicates that the calibrated value is reasonable, although it can only be treated as a precursor to more rigorous cross-checking of the calibration. 

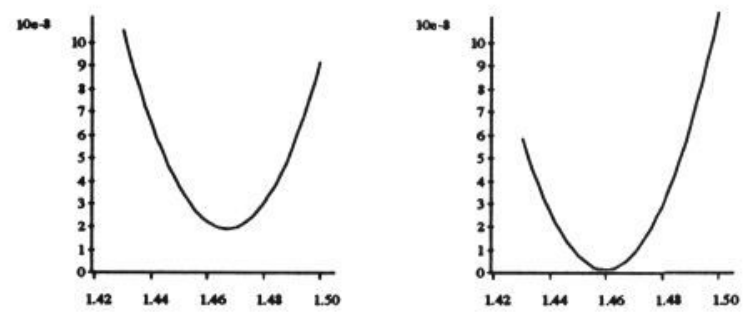

Figure 10: Left and right cameras - a plot of the residual from the computation of the concurrency of lines at the principal point, against aspect ratio. The minimum of the graph provides the estimate of the actual aspect ratio.

\begin{tabular}{|l|c|c|}
\hline Image number & Left camera focal length & Right camera focal length \\
\hline 1 & 267.7 & 264.3 \\
2 & 262.8 & 266.6 \\
3 & 261.5 & 266.6 \\
4 & 262.6 & 268.0 \\
5 & 270.0 & 266.2 \\
\hline Mean & 265 & 265 \\
Standard deviation & 4 & 1 \\
\hline
\end{tabular}

Table 1: Measurement of focal length in pixels. A focal length of 265 pixels is about $6 \mathrm{~mm}$ (using the pixel size from the camera specification).

\section{Evaluation and Conclusion}

As shown in Section 5, the constraint lines which are used to compute the principal point are reasonably close to concurrency, with an error area of about \pm 2.5 pixels in the worst case (the left carrera). The values of focal length, one from each image, show only small variation.

The results are for two short focal length wide-angle cameras. We have found that the method degrades in longer focal length cameras. This is probably because it relies on perspective effects, and these decrease as the focal length increases.

Finally, regarding the application of the method, note that the processing has no particular requirement for a symmetrical calibration pattern like the one used - the minimum requirement on the data is in fact four points in a known configuration on a

\begin{tabular}{|l|l|l|}
\hline Parameter & Left camera & Right camera \\
\hline Principal point & $(127,122)$ & $(129,124)$ \\
Focal length & 265 & 265 \\
Aspect ratio & 1.466 & 1.460 \\
\hline
\end{tabular}

Table 2: Summary of results. 
plane (the actual scale of the configuration is immaterial). This raises the possibility of extending the method to work in natural scenes - in indoor scenes, for instance, where the four corners of doorways or windows of known configuration would provide one way of obtaining the required input data. Calibrating from a natural scene is increasingly desirable with the advent of active cameras which can continually change focus and zoom - clearly though, there is a huge gulf between obtaining results using a calibration plane as presented here and the ability to calibrate from a natural scene.

\section{Acknowledgements.}

Thanks to Bill Triggs for useful insights, to Andrew Zisserman and Charlie Rothwell for their help with projective geometry, and to Han Wang for his tape calibration results.

\section{References}

[1] P.A. Beardsley. The correction of radial distortion in images. Technical report 1896/91, Department of Engineering Science, University of Oxford, 1991.

[2] P.A. Beardsley, D.W. Murray, and A.P. Zisserman. Camera calibration using multiple images. In Proc. 2nd European Conf. on Computer Vision, pages 312-320. Springer-Verlag, 1992.

[3] B. Caprile and V. Torre. Using vanishing points for camera calibration. Int. Journal of Computer Vision, pages 127-140, 1990.

[4] O.D. Faugeras and G. Toscani. The calibration problem for stereo. Proc. Conf. Computer Vision and Pattern Recognition, pages 15-20, 1986.

[5] C.A. Rothwell, A. Zisserman, C.I. Marinos, D.A. Forsyth, and J.L. Mundy. Relative motion and pose from arbitrary plane curves. Image and Vision Computing, 10(4):250-262, 1992.

[6] J.G. Semple and G.T. Kneebone. Algebraic projective geometry. Oxford University Press, 1952.

[7] R.Y. Tsai. An efficient and accurate camera calibration technique for $3 \mathrm{~d}$ machine vision. In Proc. IEEE CVPR, 1986.

[8] L. Wang and W. Tsai. Computing camera parameters using vanishing line information from a rectangular parallelepiped. Machine Vision and Applications, 3:129-141, 1990.

\section{A. Determining a Projectivity}

Figure 11(a) shows the layout of the calibration plane used in the experiments. Figure 11(b) shows the typical appearance of this calibration plane in an image, with distortion due to perspective effects. This appendix describes how to determine a linear transformation, a projectivity, which maps corresponding features between Figures 11(a) and (b).

When working with projectivities, points and lines are represented in homogeneous coordinates. Let the vertices of the calibration squares in the image be represented by $\left(x_{1}, y_{1}, 1\right) \ldots\left(x_{n}, y_{n}, 1\right)$ where $(x, y)$ is the image position in pixels and the third component is set to 1 . Let the corresponding vertices in a coordinate frame based on the calibration plane (hereafter, just the "calibration frame") be $\left(X_{1}, Y_{1}, 1\right) \ldots\left(X_{n}, Y_{n}, 1\right)$ where $(X, Y)$ are Euclidian coordinates and the third component is again set to 1 . 
(a)

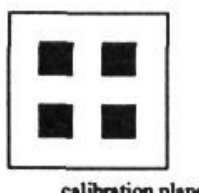

(b)

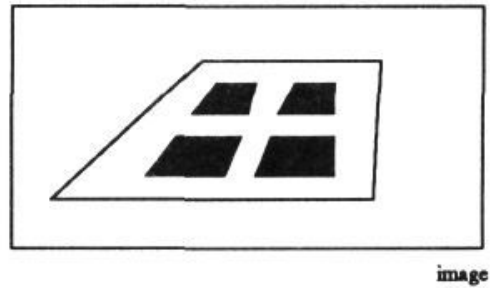

Figure 11: (a) A fronto-parallel view of the calibration plane (the plane used in the actual experiments had 4-by-4 squares). (b) The calibration plane as it appears in a typical image, with distortion due to perspective.

The choice of origin and axes for the calibration frame is arbitrary, but it is convenient to align the $x-$ and $y$-axes with the horizontal and vertical directions of the calibration squares. Note that there is no requirement here that the calibration pattern consists of squares.

The projectivity is determined by a method in [5]. Each vertex in the image is related to its corresponding point in the calibration frame by

$$
\left[\begin{array}{c}
X_{i} \\
Y_{i} \\
1
\end{array}\right]=k_{i}\left[\begin{array}{lll}
\alpha_{11} & \alpha_{12} & \alpha_{13} \\
\alpha_{21} & \alpha_{22} & \alpha_{23} \\
\alpha_{31} & \alpha_{32} & 1
\end{array}\right]\left[\begin{array}{c}
x_{i} \\
y_{i} \\
1
\end{array}\right]
$$

where the matrix $\alpha$ is the required projectivity, $\left(x_{i}, y_{i}, 1\right)$ and $\left(X_{i}, Y_{i}, 1\right)$ are corresponding points in the image and calibration frame respectively, and $k_{i}$ is a scale factor which varies with each particular pair of points. Eliminating $k_{i}$ from the three equations present in matrix equation (1) leaves two linear equations. Thus, given four pairs of points, there are eight linear equations in the eight unknowns $\alpha_{11} \ldots \alpha_{32}$, and $\alpha$ can be found ${ }^{3}$. Given $n$ points, there are $(n * 2)$ equations in the eight unknowns, and a method such as the pseudo-inverse can be used to find a solution.

It is shown in [6] that if $\alpha$ maps points from the image to the calibration frame, then $\alpha^{-1}$ is guaranteed to exist and maps points from the calibration frame to the image, $\alpha^{-\top}$ (the inverse transpose of $\alpha$ ) maps lines from the image to the calibration frame, and $\alpha^{\top}$ maps lines from the calibration frame to the image.

\footnotetext{
${ }^{3}$ The condition for linear independence of the equations is that no three of the four points are collinear.
} 